\title{
METODOLOGIA PARA CERTIFICAÇÃO DE PADRÕES SECUNDÁRIOS PARA ANÁLISES QUÍMICAS EM AMOSTRAS DE MINÉRIO DE FERRO *
}

Juliana Cecília Costa Ribeiro Vieira ${ }^{1}$ Luiz Paulo Serrano ${ }^{2}$

\section{Resumo}

Na Mina de Serra Azul da ArcelorMittal em Itatiaiuçu Minas Gerais há toda uma sistemática de controle dos processos produtivos desde a lavra, planta de concentração, estocagem, transporte ao pátio de carregamento, avaliação das características de qualidade no embarque em composições ferroviárias e assistência ao cliente no embarque do produto para a destinação final. As principais análises químicas (\%) realizadas são: $\mathrm{Fe}, \mathrm{SiO}_{2}, \mathrm{Al}_{2} \mathrm{O}_{3}, \mathrm{P}, \mathrm{Mn}, \mathrm{TiO}_{2}, \mathrm{CaO}, \mathrm{MgO}$ e Perda Por Calcinação(PPC). Essas análises são padronizadas por normas técnicas ABNT ISO tanto para atender ao mercado interno quanto ao mercado externo. Não há como garantir a qualidade das análises se não houver uma certificação da qualidade dos padrões de referência de forma a calibrar equipamentos de análise, verificar e controlar metodologias e treinar e avaliar os analistas. A produção na Mina de Serra Azul é um processo dinâmico em constante evolução e requer uma gama grande de padrões de referência com características próprias que o mercado de material de referência não tem condições de suprir. A ArcelorMittal partiu então para desenvolver uma metodologia simplificada, mas eficiente com base em critérios estatísticos consagrados para produzir seus próprios materiais de referência secundários (ou internos) de forma a atender a sua própria demanda deste tipo de material.I

Palavras-chave: Material de Referência; Estatística; Minério de Ferro.

\section{METHODOLOGY FOR PREPARATION OF SECONDARY REFERENCE MATERIAL FOR CHEMICAL ANALYSIS IN IRON ORES.}

\begin{abstract}
There is no way to guarantee the chemical quality of mining production if there is no certification of the quality of the reference standards. At the Serra Azul Mine, operated by ArcelorMittal in Itatiaiuçu Minas Gerais, there is a whole system of control of the production processes from the mining to the assistance to the customer in the shipment of the product to the final disposal. The chemical analyzes are standardized by technical standards ABNT and ISO. Production at the Serra Azul Mine requires a large range of benchmarks with its own characteristics that the reference material market can not afford. For this reason, ArcelorMittal has developed a simplified but efficient methodology based on established statistical criteria to produce its own secondary (or internal) reference materials to meet its own demand for this type of material.
\end{abstract}

Keywords: Reference Materials; Statistics; Iron Ore.

1 Graduada em Ciência Farmacêuticas e Técnica em química Industrial, supervisora de laboratório na ArcelorMittal Mineração Serra Azul, membro da Comissão de Estudos de Amostragem do CB41 Comitê Brasileiro de Minério de Ferro da ABNT, Itatiaiuçu, Minas Gerais, Brasil. 
2 Engenheiro químico, Bacharel em estatística, membro da Comissão de Estudos de Amostragem do CB41 Comitê Brasileiro de Minério de Ferro da ABNT e Consultor de métodos estatísticos para mineração, Belo Horizonte, Minas Gerais, Brasil. 


\section{INTRODUÇÃO}

Normalmente o processo de certificação de materiais de referência faz uso de programas interlaboratoriais com a participação de vários laboratórios de indiscutível competência em análise química de minérios sob a coordenação centralizada de uma instituição que prepara e envia as amostras para os testes e avalia os resultados dos participantes emitindo um certificado deste material de referência que é usado não só pelos participantes como por todas as empresas do ramo. No Brasil o principal organismo certificador é o Instituto de Pesquisas Tecnológicas (IPT) de São Paulo, que já produziu inúmeros trabalhos neste campo e preparou alguns padrões de minério de ferro. Entretanto este é um procedimento oneroso, demorado e que não atende as necessidades do processo de produção da ArcelorMittal que desenvolveu uma metodologia adequada a suas necessidades trabalhando num programa intralaboratorial utilizando seu quadro técnico e demais recursos próprios.

\subsection{PREMISSAS BÁSICAS}

Aleatorização: Tem por objetivo minimizar a possibilidade de que haja tendência nas execuções e entre os executantes.

Repetição: A repetição é indispensável para a comparação dos dados. Evidentemente, quanto maior o número de repetições mais confiável tende a ser a avaliação. No entanto, há formas de se definir um número mínimo de repetições para se atingir um determinado nível de confiança desejável.

Controle local (supervisão): O experimento deve ser conduzido de forma a avaliar as condições impostas ao planejamento e não o aspecto gerencial do controle de qualidade no dia a dia, ou seja, é preciso retirar toda e qualquer pressão sobre o executante.

Experiência profissional: É fundamental a avaliação prévia dos resultados. A Estatística é apenas uma ferramenta de auxílio à decisão, mas a experiência profissional do "pesquisador/dono" dos dados é tão importante quanto os números gerados com os testes estatísticos. O importante é criar métricas que possam aferir continuamente a qualidade dos resultados dos laboratórios.

\section{MATERIAIS E MÉTODOS}

\subsection{Planejamento}

Para realizar uma avaliação da qualidade dos resultados de análises intra/interlaboratórios, é necessário ser extremamente criterioso no desenvolvimento de um experimento, desde o seu planejamento até a sua execução, para que os objetivos traçados não sejam comprometidos por problemas na sua condução, com perda de tempo, recursos financeiros e humanos.

As amostra de minério para fins de serem certificadas como padrões internos, devem ser preparada com um rigor muito grande, a fim de garantir a qualidade do padrão. O Ideal é que se prepare aproximadamente $2,0 \mathrm{~kg}$ de material.

A amostra deve ser pulverizada e peneirada, não podendo ter não mais que $5 \%$ material retido na peneira $0,106 \mathrm{~mm}$.

Após a pulverização a amostra é homogeneizada por 30 minutos em um homogeneizador em $\mathrm{Y}$ e depois ela é quarteada em quarteador rotativo. 
É verificada a homogeneidade do material, através de analises químicas e só depois de aprovadas é realizada a certificação dos padrões.

Cada um dos 7 analistas recebeu 5 amostras teste, totalizando 35 resultados para cada execução dos ensaios de Fe (Via Úmida (VU) - Norma ABNT ISO 2597-2), $\mathrm{SiO}_{2}, \mathrm{Al}_{2} \mathrm{O}_{3}, \mathrm{P}, \mathrm{Mn}, \mathrm{CaO}, \mathrm{TiO}_{2}, \mathrm{MgO}$ (Via Espectrometria de Fluorescência de Raios $\mathrm{X}$ ) e Perda Por Calcinação. Neste trabalho apresentaremos apenas os resultados para Fe.

\subsection{Modelagem do experimento}

\subsubsection{Apresentação dos Resultados}

Os resultados das análises realizadas serão dispostos conforme a tabela 01 .

Tabela 01 - Resultados das Análises.

\begin{tabular}{c|c|c|c|c|c|c|c}
\hline \multirow{2}{*}{ Amostras } & \multicolumn{7}{|c}{ Laboratoristas } \\
\cline { 2 - 7 } & L1 & L2 & L3 & L4 & L5 & L6 & L7 \\
\hline A1 & Y & Y & Y & Y & Y & Y & Y \\
\hline A2 & Y & Y & Y & Y & Y & Y & Y \\
\hline A3 & Y & Y & Y & Y & Y & Y & Y \\
\hline A4 & Y & Y & Y & Y & Y & Y & Y \\
\hline A5 & Y & Y & Y & Y & Y & Y & Y \\
\hline
\end{tabular}

\subsubsection{Teste de Homogeneidade}

Existem métodos estatísticos que são utilizados para avaliação da homogeneidade de minérios finos. O método aplicado é análise de Variância - ANOVA - "TwoWay" para dois fatores de variabilidade - Laboratoristas e Amostras. Esta é um técnica para teste de médias, na qual as variabilidades associadas a determinadas fontes podem ser isoladas e estimadas.

Para validar a homogeneidade das amostras, o teste é realizado apenas para o elemento Ferro, pois é o elemento de maior percentual constituinte e de maior importância para a Mineração.

As variáveis são calculadas conforme a tabela 02, onde:

Tabela 02 -"ANOVA - TwoWay" para dois fatores de variabilidade - Laboratoristas e Amostras

\begin{tabular}{|c|c|c|c|c|c|}
\hline Fatores & SQ & GL & QM & Fcalc/.tab & ValorP \\
\hline Labs & $S Q L=\frac{\sum_{i=1}^{L} L^{2}}{A}-C_{\xi}$ & $V_{L}=\mathrm{L}-1$ & $Q_{L}=S Q_{L} / V_{L}$ & $F_{C L}=Q M_{L} / Q_{R}$ & $V p_{L}$ \\
\hline Amostras & $S Q A=\frac{\sum_{j=1}^{A} A^{2}}{L}-C$ & $V_{A}=\mathrm{A}-1$ & $\mathrm{QM}_{\mathrm{A}}=S \mathrm{SQ}_{\mathrm{A}} / V_{A}$ & $F_{C A}=Q_{A} / Q_{R}$ & $V p_{A}$ \\
\hline Resíduos & $S Q_{R}=S Q_{T}-S Q_{A}-S Q_{L}$ & $V_{R}=(\mathrm{L}-1)^{\star}(\mathrm{A}-1)$ & $\mathrm{QM}_{\mathrm{R}}=S \mathrm{SQ}_{\mathrm{R}} / V_{R}$ & Ftabı & \\
\hline Total & $S Q T=\sum_{k=1}^{L \gtrless A} y^{2}-C$ & $V_{T}=\left(L^{*} A\right)-1$ & & FtabA $_{A}$ & \\
\hline
\end{tabular}


$\mathrm{L}$ : número de analistas

A: número de amostras

Y: resultado de cada análise

O valor de C, é dado pela equação 1 .

$$
C=\frac{\left(\sum y\right)^{2}}{L * A}
$$

Na tabela de distribuição de probabilidade de Fisher-Snedecor (Montgomery1997[9]), para nível de significância de 5\% procurar:

Para: $\quad$ Ftabı usar os valores de $V_{L}$ e $V_{R}$

FtabA usar os valores de $V_{A}$ e $V_{R}$

Os valores de $V p_{\llcorner}$e $V p_{A}$ são obtidos por cálculo numérico diretamente da função de distribuição de probabilidade de Fisher - Snedecor.

A Hipótese da homogeneidade é aceita se $F c_{A}<F_{t a b}$ e $V p_{A}>0,05$.

\subsubsection{Análise descritiva do conjunto de dados}

Foram determinados para o conjunto de 35 resultados, as estatísticas, conforme a tabela 03 abaixo.

\begin{tabular}{l|c|c|c|c|c}
\hline \multicolumn{6}{c|}{ Tabela 03 - Análise Descritiva dos Dados } \\
Mínimo & Mediana & Média & Máximo & $\begin{array}{c}\text { Amplitude Máxima } \\
\text { (Rmáx) }\end{array}$ & Variância \\
\hline \hline & $\begin{array}{c}\text { Coeficiente de } \\
\text { Variação (CV) }\end{array}$ & $\begin{array}{c}\text { № } \\
\text { Réplica }\end{array}$ & $\begin{array}{c}\text { № } \\
\text { Analista }\end{array}$ & Dados Totais \\
\hline
\end{tabular}

As fórmulas para os cálculos não serão apresentadas por serem muito utilizadas e de fácil entendimento.

\subsubsection{Avaliação de 'outliers' e simetria do conjunto de dados Grubs e BoxPlot}

Antes de se efetuar a avaliação e interpretação de uma série de resultados obtidos em ensaios ou calibrações, ou de se efetuar o cálculo da incerteza, é necessário verificar a existência de valores que possam,eventualmente, serem considerados como dispersos.

Observações dispersas são valores anormalmente baixos ou altos, que aparecem como anomalias em uma série de dados.

Os testes mais comuns para determinar a presença de dados dispersos são os testes de Cochran, Dixon e de Grubbs. Vamos utilizar neste trabalho os testes de Cochran e Grubbs.

\section{Teste de Grubbs}

A avaliação dos dados é feita comparando o valor tabelado com os valores calculados. Para se proceder à avaliação, os dados devem ser ordenados em ordem crescente, após deve-se aplicar os cálculos conforme as equações 2 e 3. 
$\mathbf{n}=$ numero de resultados

$$
\begin{gathered}
G_{n}=\frac{X_{1}-X_{n}}{S} \\
G_{p}=\frac{X_{p}-X_{n}}{S}
\end{gathered}
$$

$\mathbf{X}_{\mathbf{n}}=$ Média dos resultados

$\mathbf{X}_{\mathbf{1}}=$ menor valor

$\mathbf{X}_{\mathbf{p}}=$ maior valor

$\mathbf{S}=$ Desvio padrão

$\mathbf{G}_{\mathbf{n}}=$ Significancia do menor valor

$\mathbf{G}_{\mathbf{p}}=$ Significancia do maior valor

Se o valor crítico tabelado $\left(G_{c}\right.$ - tabela C.1, ABNT $\left.14597^{[1]}\right)$ for maior do que o valor calculado para o menor valor $\left(\boldsymbol{G}_{n}\right)$, isto é $\boldsymbol{G}_{\boldsymbol{C}}>\boldsymbol{G}_{\boldsymbol{n}}$, o menor valor dos resultados não será considerado discrepante.

Se o valor crítico tabelado $\left(G_{c}\right.$ - tabela C.1, ABNT $\left.14597^{[1]}\right)$ for maior do que o valor calculado para o maior valor $\left(\boldsymbol{G}_{\boldsymbol{p}}\right)$, isto é $\boldsymbol{G}_{\boldsymbol{C}}>\boldsymbol{G}_{\boldsymbol{p}}$, o maior valor calculado dos resultados não será considerado discrepante.

Caso seja identificado algum valor discrepante, o responsável pela execução do experimento deve procurar verificar se há alguma causa identificável e corrigí-la antes de continuar, caso contrário deve manter o resultado.

\section{Box Plot}

A avaliação do Diagrama de Caixa (Box Plot), nos permite verificar se os dados têm uma distribuição simétrica e se há presença de "outliers".

Caso a avaliação apresente algum valor outlier, deve-se discutir com o analista e tentar encontrar as possíveis causas, a análise pode ser refeita. Não é possível excluir apenas uma análise, no caso seria necessário excluir o analista, o que não é conveniente em se tratando de um trabalho intralaboratorial.

\subsubsection{Avaliação dos resultados médios por analistas}

Efetuar a analise dos resultados médios, por analista, calcular a média e variância e apresentar os resultados, conforme tabela 4.

Tabela 4 : Resultados médio dos analistas

\begin{tabular}{c|c|c}
\hline Analista $(\boldsymbol{p})$ & Médias & Variâncias \\
\hline $\mathrm{L} 1$ & $\mathrm{X}_{\mathrm{m}}$ & $\mathrm{S}^{2} \mathrm{~L} 1$ \\
\hline $\mathrm{L} 2$ & $\mathrm{X}_{\mathrm{m}}$ & $\mathrm{S}^{2} \mathrm{~L} 2$ \\
\hline $\mathrm{L} 3$ & $\mathrm{X}_{\mathrm{m}}$ & $\mathrm{S}^{2} \mathrm{~L} 3$ \\
\hline $\mathrm{L} 4$ & $\mathrm{X}_{\mathrm{m}}$ & $\mathrm{S}^{2} \mathrm{~L} 4$ \\
\hline $\mathrm{L} 5$ & $\mathrm{X}_{\mathrm{m}}$ & $\mathrm{S}^{2} \mathrm{~L} 5$ \\
\hline $\mathrm{L} 6$ & $\mathrm{X}_{\mathrm{m}}$ & $\mathrm{S}^{2} \mathrm{~L} 6$ \\
\hline $\mathrm{L} 7$ & $\mathrm{X}_{\mathrm{m}}$ & $\mathrm{S}^{2} \mathrm{~L} 7$ \\
\hline
\end{tabular}




\subsubsection{Valores discrepantes dos analistas (Teste de Grubbs)}

Deve-se proceder ao teste de Grubbs, conforme descrito no item 2.2.4, utilizando os valores médios de cada analista, conforme as equações 2 e 3.

\subsubsection{Variância dos analistas (COCHRAN)}

\section{Teste de Cochran:}

A avaliação dos dados é feita comparando o valor tabelado (tabela B.1, ABNT $14597^{[1]}$ ) com o valor calculado, conforme a equação 4.

$$
C_{c}=\frac{S_{\text {Max }}^{2}}{S_{\text {Total }}^{2}}
$$

$S^{2} \max =$ Maior variância.

$\mathrm{S}^{2}$ Total $=$ Soma de todas as variâncias.

$\mathrm{C}_{\mathrm{C}}$ é o valor Calculado

$\mathrm{C}_{\mathrm{tab}}$ é um valor tabelado

Se o valor calculado for menor que o valor tabelado, resultado homogêneo, prosseguir com a avaliação dos resultados. Se o valor calculado for maior que o valor tabelado, resultado heterogêneo, avaliar as possíveis causas da heterogeneidade dos dados do analista com a maior variância.

Caso os valores sejam rejeitados nas duas ou em uma das avaliações, 2.2.6 / 2.2.7, deve-se discutir com o analista e tentar encontrar as possíveis causas, a análise pode ser refeita. Não é possível excluir apenas uma análise, no caso seria necessário excluir o analista, o que não é conveniente em se tratando de um trabalho intralaboratorial.

\subsubsection{Análise de variância com um fator de variabilidade (ANOVA)}

Utilizou-se como ferramenta de análise dos dados, a Análise de Variância com um fator ou Delineamento Inteiramente Casualizado - DIC (ANOVA "OneWay", [Montgomery 1997[9]]. A avaliação é feita conforme a tabela 5.

Neste artigo, vamos descrever apenas os resultados obtidos para Fe VU, os parâmetros para os demais elementos foram tratados com a mesma metodologia.

Tabela 5- Levantamento dos dados para ANOVA

\begin{tabular}{|c|c|c|c|c|c|c|c|}
\hline Laboratoristas (p) & \multicolumn{5}{|c|}{ Réplicas (n) } & Total Fator 0 & Media geral \\
\hline L1 & $\mathrm{Xi}$ & $\mathrm{Xi}$ & $\mathrm{Xi}$ & $\mathrm{Xi}$ & $\mathrm{Xi}$ & & \multirow[b]{2}{*}{$\bar{X}$} \\
\hline $\mathrm{L} 2$ & $\mathrm{Xi}$ & $\mathrm{Xi}$ & $\mathrm{Xi}$ & $\mathrm{Xi}$ & $\mathrm{Xi}$ & & \\
\hline L3 & $X i$ & $\mathrm{Xi}$ & $\mathrm{Xi}$ & $\mathrm{Xi}$ & $\mathrm{Xi}$ & & \\
\hline L4 & $\mathrm{Xi}$ & $\mathrm{Xi}$ & $\mathrm{Xi}$ & $\mathrm{Xi}$ & $\mathrm{Xi}$ & & \\
\hline L5 & $\mathrm{Xi}$ & $\mathrm{Xi}$ & $\mathrm{Xi}$ & $\mathrm{Xi}$ & $\mathrm{Xi}$ & & \\
\hline L6 & $\mathrm{Xi}$ & $\mathrm{Xi}$ & $\mathrm{Xi}$ & $\mathrm{Xi}$ & $\mathrm{Xi}$ & & \\
\hline $\mathrm{L7}$ & $\mathrm{Xi}$ & $\mathrm{Xi}$ & $\mathrm{Xi}$ & $\mathrm{Xi}$ & $\mathrm{Xi}$ & & \\
\hline
\end{tabular}


$n=$ numero de réplicas

Total fator $\mathrm{O}=$ a soma dos $\mathrm{n}$ resultados de cada $\mathrm{p}$ nível $p=$ numero de analistas

$X i=$ cada resultado

A média Geral é calculada conforme a equação 5.

$$
\bar{X}=\frac{\sum X_{i}}{\sum n}
$$

Para análise da variância é necessário o cálculo de alguns fatores que serão utilizados na tabela 06, eles são representados pelas equações 6,7 e 8 .

$$
\begin{array}{r}
A=\frac{\left(\sum X_{i}\right)^{2}}{n p} \\
B=\frac{\sum(\text { Total fator } 0)^{2}}{n} \\
C=\sum X_{i}^{2}
\end{array}
$$

A análise de variância dá subsídios para determinação da repetibilidade e

\begin{tabular}{|c|c|c|c|c|}
\hline Fator & $\begin{array}{c}\text { Soma dos } \\
\text { Quadrados } \\
\text { (SQ) }\end{array}$ & $\begin{array}{c}\text { Grau de } \\
\text { Liberdade ( })\end{array}$ & $\begin{array}{c}\text { Média da Soma } \\
\text { dos Quadrados } \\
\text { (QM) }\end{array}$ & $\begin{array}{c}\text { Estimadores das } \\
\text { variâncias }\left(\mathbf{S}^{2}\right)\end{array}$ \\
\hline $\begin{array}{c}\text { Analista } \\
(0)\end{array}$ & $S Q_{0}=B-A$ & $V_{0}=p-1$ & $\mathrm{QM}_{0}=\mathrm{SQ}_{0} / \mathrm{V}_{0}$ & $\mathrm{~S}^{2} 0=\left(\mathrm{QM}_{0}-\mathrm{QM}_{\mathrm{r}}\right) / n$ \\
\hline residual $_{(\mathrm{r})}$ & $S Q_{r}=C-B$ & $v_{r}=p(n-1)$ & $\mathrm{QM}_{\mathrm{r}}=\mathrm{S} \mathrm{Q}_{\mathrm{r}} / \mathrm{V}_{\mathrm{r}}$ & $S^{2} r=Q M_{r}$ \\
\hline Total & $S_{Q}=C-A$ & $V_{\top}=(p n)-1$ & & EQM \\
\hline
\end{tabular}
reprodutibilidade, além de avaliar a qualidade dos resultados dos analistas.

O Fator Analista é o fator de variabilidade estudado. A tabela 6 mostra como devem ser feitos os cálculos.

\subsubsection{Erro médio quadrático (EQM)}

É uma métrica que permite avaliar a qualidade dos padrões secundários produzidos, e quanto mais próximos de zero melhor. Utilizada para monitoramento de cada elemento, na confecção dos padrões é definida pela equação 9.

$$
E Q M=\sqrt{\frac{\sum_{i=1}^{N}(\text { Resíduos })^{2}}{N}}
$$

Resíduos $=0$ resultado de cada réplica do analista menos a média do analista.

Essa pode ser uma métrica para comparação futura da modelagem. Por experiência, sabe-se que $E Q M<0,20 \%$ para $F e$ é satisfatório, mas quanto menor for o $E Q M$, melhor, já que o ideal é que o erro aleatório seja o menor possível (próximo de zero). 


\subsubsection{Comparações múltiplas DMS - t de Fischer}

Teste $\mathbf{t}$ de Fisher: é a determinação da Diferença Mínima Significativa (DMS), a qual apresenta a diferença estatisticamente tolerável na comparação entre duas médias, calculado pela equação 10 .

$t_{(N, \alpha)}$ é a variável da distribuição $t$ de Students

$$
D M S=t_{(N, \alpha)} * \sqrt{\frac{2 * Q M_{r}}{n}}
$$

$Q M_{r}$ é determinado na tabela 06.

Se a diferença entre as médias dos analistas estiver acima do valor de DMS, encontrado, os analistas devem ser avaliados.

\subsubsection{Determinação de repetibilidade $(r)$ e reprodutibilidade $(R)$}

Repetibilidade $(\boldsymbol{r})$ é a tolerância entre duas medidas realizadas pelo mesmo analista $(a=2)$.

Reprodutibilidade $(\boldsymbol{R})$ é a tolerância dentre duas medidas realizadas por analistas diferentes $(a=2)$. Os cálculos devem ser realizados conforme as equações 11,12 , 13 e 14 .

$$
\begin{array}{r}
S_{r}=\sqrt{\mathrm{QM}_{\mathrm{r}}} \\
S_{R}=\sqrt{S_{0}^{2}+S_{r}^{2}} \\
r=Z_{\alpha} * \sqrt{a} * S_{r} \\
R=Z_{\alpha} * \sqrt{a} * S_{R}
\end{array}
$$

Onde $Z_{\alpha}$ é uma variável padrão que determina o nível de confiança a ser adotado, para um nível de $95 \%$ de confiança o valor utilizado é 1,96. Esses valores já são tabelados ${ }^{[5]}$.

\subsubsection{Estimativa da exatidão}

A exatidão fornece uma visão da qualidade dos resultados médios dos analistas em relação ao valor da média do Material de Referência Certificado (MRC) ou mesmo em relação a um padrão interno a critério do Coordenador do Experimento. Para essa métrica utilizamos o Índice de Score $(Z)$, que é calculado conforme a equação 15.

$\overline{\mathrm{X}}_{\mathrm{L}}=$ médida de cada um dos analistas

$$
\mathrm{z}=\frac{\left|\overline{\mathrm{X}}_{\mathrm{L}}-\mathrm{X}_{\mathrm{MRC}}\right|}{\mathrm{S}_{\mathrm{MRC}}}
$$

$\mathrm{X}_{\mathrm{MRC}}=$ valor de referência ou valor médio do material

$\mathrm{S}_{\mathrm{MRC}}=$ Desvio padrão do material de referência ou dos valores médios.

A avaliação é feita conforme abaixo:

$z \leq 2$ pode-se considerar o resultado satisfatório.

$2<z \leq 3$, o resultado é considerado duvidoso e.

$z>3$, o resultado é considerado não satisfatório ou insatisfatório. 
RESULTADOS E DISCUSSÃO

\subsection{Apresentação dos Resultados}

Os resultados obtidos nas análises estão apresentados na tabela 07.

Tabela 07 - Resultados das Análises

\begin{tabular}{c|c|c|c|c|c|c|c}
\hline \multirow{2}{*}{ Amostras } & \multicolumn{7}{|c}{ Laboratoristas } \\
\cline { 2 - 8 } & L1 & L2 & L3 & L4 & L5 & L6 & L7 \\
\hline A1 & 46,422 & 46,257 & 46,406 & 46,260 & 46,204 & 46,383 & 46,190 \\
\hline A2 & 46,438 & 46,315 & 46,313 & 46,290 & 46,371 & 46,415 & 46,230 \\
\hline A3 & 46,397 & 46,380 & 46,367 & 46,325 & 46,319 & 46,442 & 46,281 \\
\hline A4 & 46,378 & 46,339 & 46,320 & 46,281 & 46,365 & 46,296 & 46,311 \\
\hline A5 & 46,399 & 46,390 & 46,327 & 46,318 & 46,362 & 46,378 & 46,401 \\
\hline
\end{tabular}

\subsection{Teste de Homogeneidade}

A tabela 08 apresenta os resultados encontrados para o teste de homogeneidades. Os valores grifados devem ser observados para validação da homogeneidade.

Tabela 08 -"ANOVA - TwoWay" para dois fatores de variabilidade Laboratoristas e Amostras

\begin{tabular}{l|c|c|c|r|r}
\hline \multicolumn{1}{c|}{ Fatores } & SQ & GL & QM & Fcalc/tab & ValorP \\
\hline Labs & 0,05967189 & 6 & 0,00995 & 3,833 & 0,008 \\
\hline Amostras & 0,01853343 & 4 & 0,00463 & 1,786 & 0,165 \\
\hline Resíduos & 0,06226697 & 24 & 0,00259 & 2,508 & \\
\hline Total & 0,14047229 & 34 & 0,00413 & 2,776 & \\
\hline
\end{tabular}

A Hipótese da homogeneidade é aceita se $F c_{A}<F_{t a b}$ e $V p_{A}>0,05$, logo há evidência da HOMOGENEIDADE das amostras.

\subsection{Análise descritiva do conjunto de dados}

A tabela 9 traz o resultado da análise descritiva dos dados.

Tabela 9: Estatística descritiva do conjunto de dados

\begin{tabular}{|c|c|c|c|c|c|c|c|c|}
\hline Min & Mediana & Média & Max & $\mathbf{R}_{\text {Máx }}$ & $\boldsymbol{\sigma}_{\text {Tot }}^{2}$ & $\boldsymbol{\sigma}_{\text {Tot }}$ & $\mathbf{C V}_{(\%)}$ & $\mathbf{N}$ \\
\hline 46,19 & 46,34 & $\mathbf{4 6 , 3 4}$ & 46,44 & 0,25 & 0,0041 & 0,064 & 0,14 & 35 \\
\hline
\end{tabular}

\subsection{Avaliação de 'outliers' e simetria do conjunto de dados - Grubbs e BoxPlot}

A figura 01, apresenta a distribuição e simetria do conjunto de dados, mostrando que não há evidência de outliers. 


$$
\begin{array}{|l|l|l|l|l|}
\boldsymbol{G}_{n}=2,320 \quad \boldsymbol{G}_{p}=1,600 \quad \boldsymbol{G}_{c}= & 2,979 \\
\hline
\end{array}
$$

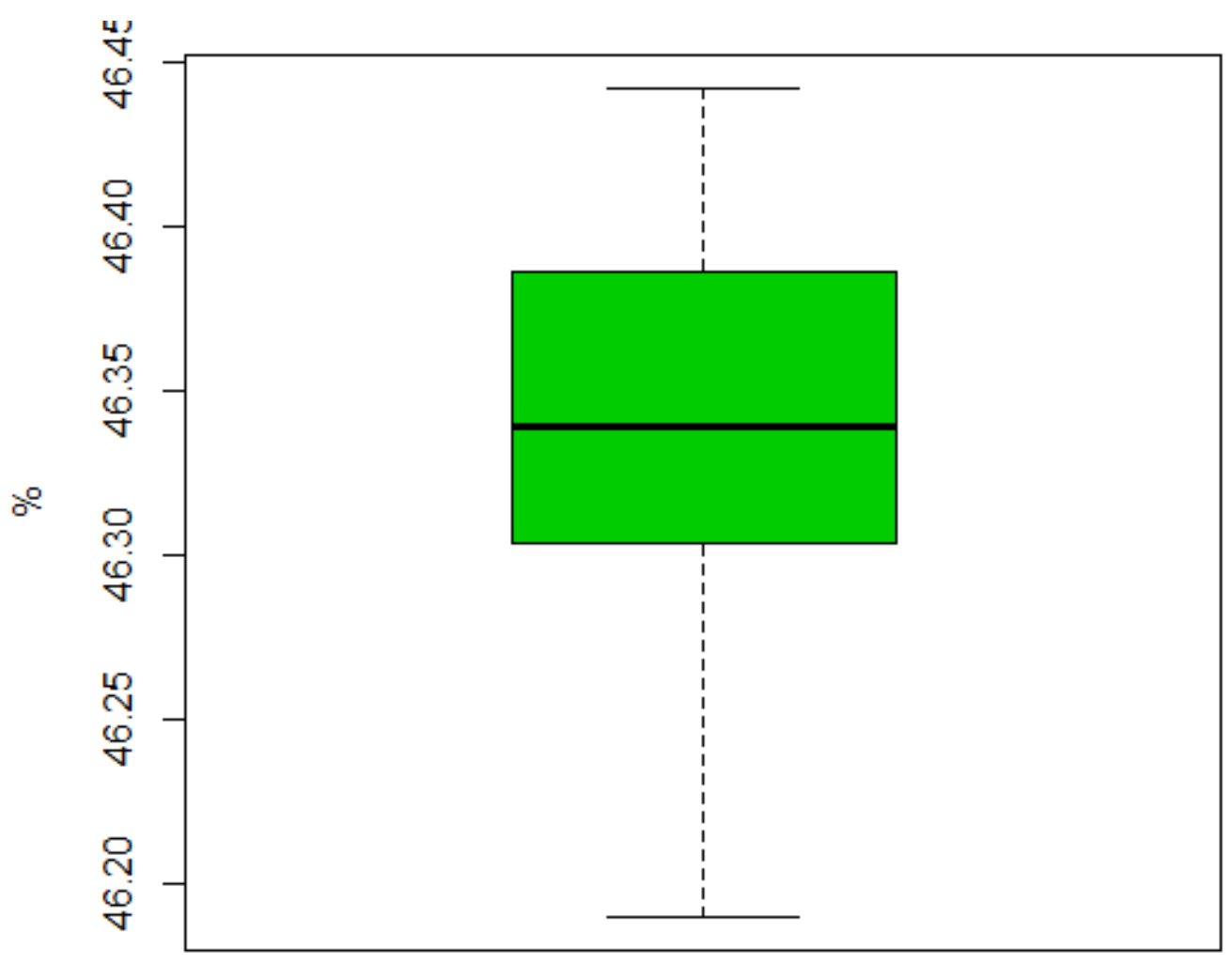

Amostras

Figura 01 - Box Plot - Avaliação Previa do Conjunto de dados

\subsection{Avaliação dos resultados médios por analistas}

A tabela 10, apresenta os resultados médios e variâncias de cada analista.

Tabela 10 : Resultados médio dos analistas

\begin{tabular}{|c|r|r|}
\hline Analista $(\boldsymbol{p})$ & \multicolumn{1}{|c|}{ Médias } & \multicolumn{1}{l|}{ Variâncias } \\
\hline L1 & $\mathbf{4 6 , 4 0 6 8}$ & $\mathbf{0 , 0 0 0 5 4 7 7}$ \\
\hline L2 & $\mathbf{4 6 , 2 9 4 8}$ & $\mathbf{0 , 0 0 0 7 1 8 7}$ \\
\hline L3 & $\mathbf{4 6 , 3 4 6 6}$ & $\mathbf{0 , 0 0 1 5 4 1 3}$ \\
\hline L4 & $\mathbf{4 6 , 3 3 6 2}$ & $\mathbf{0 , 0 0 2 8 8 5 7}$ \\
\hline L5 & $\mathbf{4 6 , 3 8 2 8}$ & $\mathbf{0 , 0 0 3 0 2 4 7}$ \\
\hline L6 & $\mathbf{4 6 , 3 2 4 2}$ & $\mathbf{0 , 0 0 4 9 3 9 7}$ \\
\hline L7 & $\mathbf{4 6 , 2 8 2 6}$ & $\mathbf{0 , 0 0 6 5 4 2 3}$ \\
\hline
\end{tabular}




\subsection{Valores discrepantes dos analistas (Teste de Grubbs)}

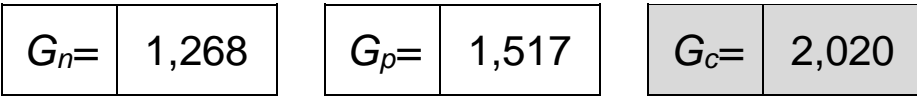

Não há evidência de outliers.

\subsection{Variância dos analistas (COCHRAN)}

\begin{tabular}{|c|c|}
\hline $\mathbf{n}$ & $\mathbf{5}$ \\
\hline $\mathbf{p}$ & $\mathbf{7}$ \\
\hline $\boldsymbol{S}^{2}$ max & $\mathbf{0 , 0 0 6 5 4 2}$ \\
\hline $\boldsymbol{S}^{2}$ Total & $\mathbf{0 , 0 2 0 2 0 0}$ \\
\hline $\boldsymbol{C}_{\boldsymbol{c}}$ & $\mathbf{0 , 3 2 4}$ \\
\hline $\boldsymbol{C}_{\text {tab }}$ & $\mathbf{0 , 3 9 7}$ \\
\hline
\end{tabular}

Há evidência da igualdade das variâncias

\subsection{Análise de variância com um fator de variabilidade (ANOVA - "OneWay")}

A tabela 11 apresenta os dados calculados para realização da analise de variância.

\begin{tabular}{|c|c|c|c|c|c|c|}
\hline Fator & $\begin{array}{c}\text { Soma dos } \\
\text { Quadrados } \\
\text { (SQ) }\end{array}$ & $\begin{array}{c}\text { Grau de } \\
\text { Liberdade } \\
\text { (V) }\end{array}$ & $\begin{array}{c}\text { Média da } \\
\text { Soma dos } \\
\text { Quadrados } \\
\text { (QM) }\end{array}$ & $\begin{array}{l}\text { Estimadores } \\
\text { das } \\
\text { variancias } \\
\left(\mathrm{S}^{2}\right)\end{array}$ & $F_{\text {calc. }}$ & $\begin{array}{c}\text { Valor } \\
\mathbf{P}\end{array}$ \\
\hline Analista (0) & 0,059672 & 6 & 0,009945 & 0,001412 & 3,446 & \\
\hline residual $(r)$ & 0,0808 & 28 & 0,002886 & 0,002886 & $F_{\text {tab }}$ & \\
\hline Total & 0,140472 & 34 & & & 2,445 & 0,011 \\
\hline
\end{tabular}

Do ponto de vista teórico, o que se tem por trás da tabela 11, é um teste de hipóteses, em que as hipóteses a serem testadas são:

$H_{0}$ : não há evidências para se rejeitar a igualdade entre os resultados médios de todos os laboratoristas.

$H_{1}$ : pelo menos um laboratorista não apresenta média igual aos demais;

Se Valor-p $<0,05$, [Hogg \& Tanis $1999{ }^{[4]}$ ], rejeita-se $H_{0}$ em favor de $H_{1}$, ao nível de significância de 5\%. Ao nível de significância de $1 \%$ (Valor-p < 0,01) não rejeitaríamos $H_{0}$. Nesse caso, se for considerado que os resultados são aceitáveis, pode-se concluir (com ressalvas) pela igualdade das médias dos laboratoristas.

Neste teste estatístico, fica evidenciado que há no mínimo um Laboratorista que apresenta média diferente dos demais, ao nível de significância de 5\%. Observe-se o gráfico da Figura 02. 


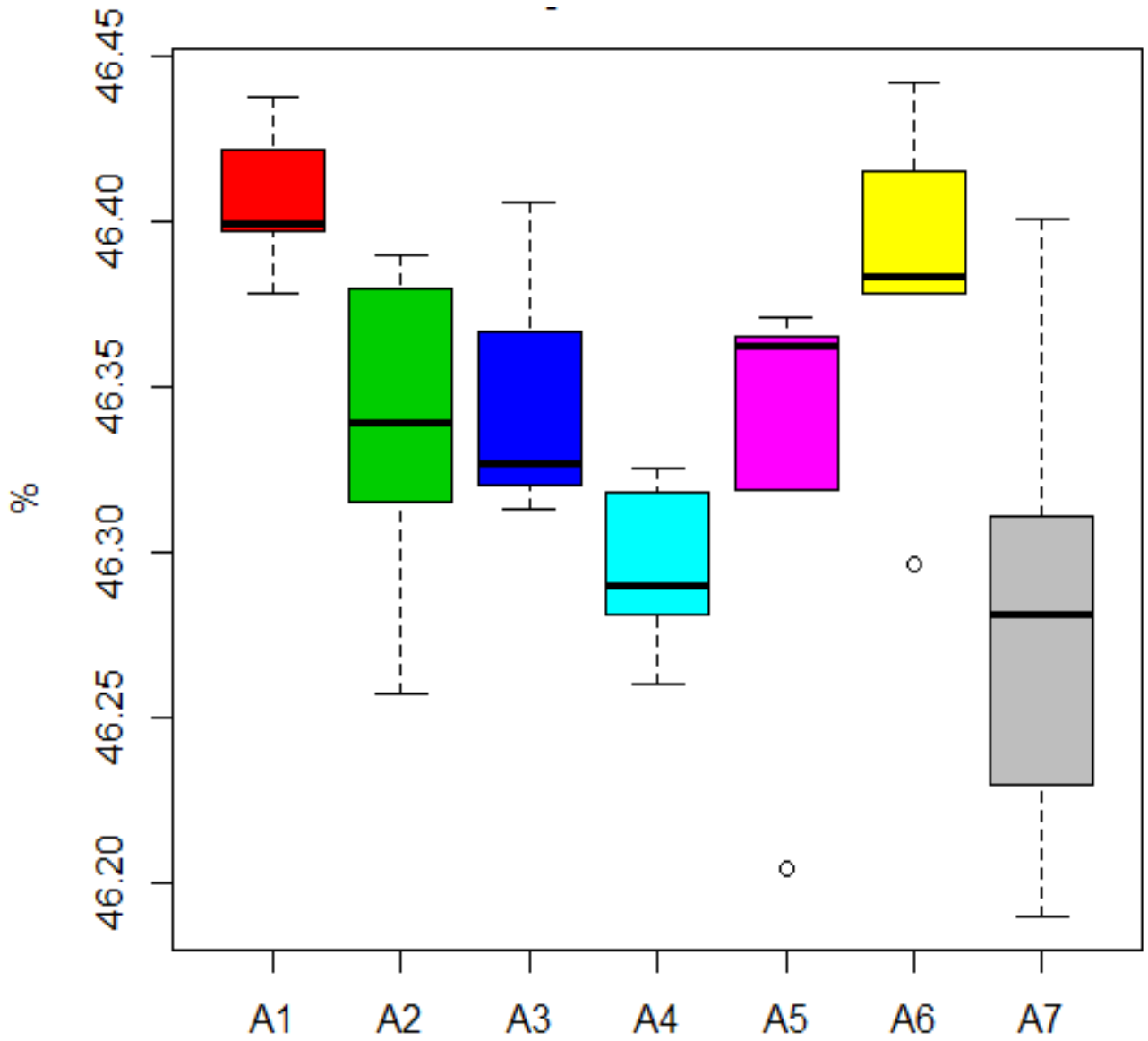

Analistas

Figura 2- BoxPlot - Avaliação dos Analistas

\subsection{Erro médio quadrático (EQM)}

$$
E Q M=0,048 \%
$$

\subsection{Comparações múltiplas DMS - t de Fischer}

Foram assinalados em vermelho as diferenças que deram acima do valor de DMS. Observamos que os laboratoristas L1, L4 e L7, apresentaram diferenças acima do DMS, porém após discussões interna foi definido que eles não seriam retirados desse programa de certificação. 


\begin{tabular}{|c|r|}
\hline Analista & Diferença \\
\hline L2-L1 & 0,071 \\
\hline L3-L1 & 0,060 \\
\hline L4-L1 & 0,112 \\
\hline L5-L1 & 0,083 \\
\hline L6-L1 & 0,024 \\
\hline L7-L1 & 0,124 \\
\hline L3-L2 & 0,011 \\
\hline L4-L2 & 0,041 \\
\hline L5-L2 & 0,012 \\
\hline L6-L2 & 0,047 \\
\hline L7-L2 & 0,053 \\
\hline
\end{tabular}

\begin{tabular}{|c|r|}
\hline Analista & Diferença \\
\hline L4-L3 & 0,052 \\
\hline L5-L3 & 0,023 \\
\hline L6-L3 & 0,036 \\
\hline L7-L3 & 0,064 \\
\hline L5-L4 & 0,029 \\
\hline L6-L4 & 0,088 \\
\hline L7-L4 & 0,012 \\
\hline L6-L5 & 0,059 \\
\hline L7-L5 & 0,041 \\
\hline L7-L6 & 0,100 \\
\hline DMS & $\mathbf{0 , 0 7 0}$ \\
\hline
\end{tabular}

\subsection{Determinação de repetibilidade $(r)$ e reprodutibilidade $(R)$}

Repetibilidade e reprodutibilidade foram calculadas para um nível de confiança de $95 \%$.

$r=0,150 \%$

$R=\mathbf{0 , 1 8 4} \%$

\subsection{Estimativa da exatidão}

\begin{tabular}{|c|c|r|}
\hline Analista (p) & Médias & \multicolumn{1}{|c|}{ Z-score } \\
\hline L1 & 46,4068 & $\mathbf{1 , 5 1 7}$ \\
\hline L2 & 46,2948 & $\mathbf{0 , 9 9 4}$ \\
\hline L3 & 46,3466 & $\mathbf{0 , 1 6 7}$ \\
\hline L4 & 46,3362 & $\mathbf{0 , 0 6 6}$ \\
\hline
\end{tabular}

\begin{tabular}{|c|r|r|}
\hline Analista (p) & \multicolumn{1}{|c|}{ Médias } & Z-score \\
\hline L5 & 46,3828 & $\mathbf{0 , 9 7 9}$ \\
\hline L6 & 46,3242 & $\mathbf{0 , 3 3 5}$ \\
\hline L7 & 46,2826 & $\mathbf{1 , 2 6 8}$ \\
\hline Média & $\mathbf{4 6 , 3 3 9}$ & \multicolumn{1}{|c}{} \\
\cline { 1 - 2 } Desvio & $\mathbf{0 , 0 4 4 6}$ & \multicolumn{1}{|c}{} \\
\cline { 1 - 2 } & \multicolumn{2}{|l}{}
\end{tabular}

Todos os analistas trabalham com exatidão aceitáveis.

\subsection{Modelo de Certificado de Análise}

Foi criado um modelo de certificado de análise. Na figura 03 apresentamos os resultados de todas as características de qualidade de interesse para o Material de Referência Interno identificado como PI. M001-JF, que possibilita ao analista verificar se as suas analises estão dentro dos parâmetros de repetibilidade, reprodutibilidade (em relação aos demais analistas) e exatidão aceitos pelo Z-Score. 


\begin{tabular}{|c|c|c|c|c|c|}
\hline \multicolumn{6}{|c|}{ CERTIFICADO DE ANÁLISE } \\
\hline \multicolumn{5}{|c|}{ PI. M001- JF } & \multirow{3}{*}{ ArcelorMittal } \\
\hline Material: Minério de & & igem: & Mina Se & & \\
\hline \multicolumn{5}{|c|}{ Material de Referencia Interno } & \\
\hline Análise (\%) & Valor Certificado & $\mathbf{r}$ & $\mathbf{R}$ & $\sigma_{M}$ & $\begin{array}{l}\text { Métodos } \\
\text { Analíticos }\end{array}$ \\
\hline Fe Total & 56,377 & 0,333 & 0,333 & 0,119 & Método Titrimétrico \\
\hline $\mathrm{SiO}_{2}$ & 12,984 & 0,263 & 0,267 & 0,100 & Método do Raios $\mathrm{X}$ \\
\hline $\mathrm{Al}_{2} \mathrm{O}_{3}$ & 2,976 & 0,101 & 0,102 & 0,038 & Método do Raios $\mathrm{X}$ \\
\hline $\mathbf{P}$ & 0,0545 & 0,0023 & 0,0024 & 0,00085 & Método do Raios $\mathrm{X}$ \\
\hline Mn & 0,0089 & 0,0014 & 0,0015 & 0,00052 & Método do Raios $\mathrm{X}$ \\
\hline $\mathrm{CaO}$ & 0,0109 & 0,0041 & 0,0041 & 0,00145 & Método do Raios $\mathrm{X}$ \\
\hline $\mathrm{TiO}_{2}$ & 0,2625 & 0,0103 & 0,0103 & 0,00364 & Método do Raios $\mathrm{X}$ \\
\hline $\mathrm{MgO}$ & 0,0110 & 0,0193 & 0,0196 & 0,00698 & Método do Raios X \\
\hline PPC & 2,9190 & 0,108 & 0,110 & 0,039 & Método Gravimétrico \\
\hline
\end{tabular}

r- Repetibilidade

R - Reprodutibilidade

$\sigma_{M}$ desvio padrão do conjunto de todas as medidas realizadas

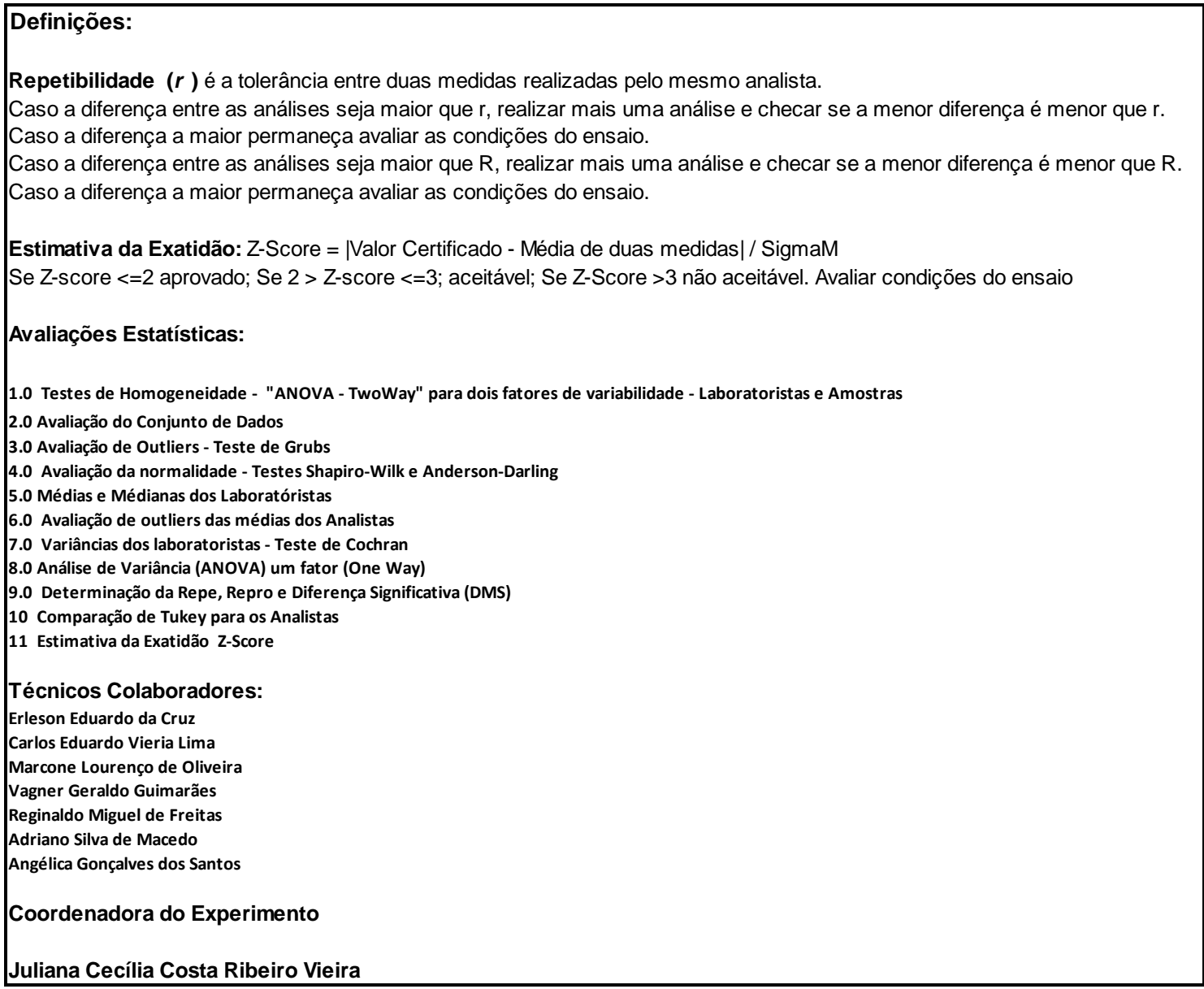




\subsection{Conclusões}

Este Material de Referência Interno foi obtido num prazo de 3 meses e vai ser usado nas operações das Minas e das Usinas Siderúrgicas do grupo ArcelorMittal em Minas Gerais.

Com a maior quantidade de padrões o laboratório vai poder reduzir o custo direto, com a compra de padrões, além de intensificar os seus controles internos. Para as usinas os padrões são confeccionados de acordo com o material utilizado por cada uma, visto que é utilizado pó prensado, assim garantimos curvas com maior número de padrões e uma precisão muito melhor e com desvios menores, a um custo muito baixo na operação dos laboratórios, além de garantir melhores resultados.

Outro ponto importante é a utilização destes dados para avaliar os analistas, verificando a necessidades de treinamento, a existência de vícios de análises, dentre outros. O mais importante é a experiência do pesquisador ("dono dos dados"), para definir métricas que possam ser usadas para o acompanhamento da evolução da qualidade do laboratório.

A análise prévia do conjunto de dados é sempre muito importante no sentido de verificar alguma inconsistência com os dados antes de dar seguimento à avaliação estatística final.

É um trabalho que demanda recursos internos e tempo, mas que traz grande retorno financeiro e de qualidade, principalmente para quem trabalha com pastilha prensada.

\section{Referências}

1- ABNT NBR ISO 14597: (2012). Programa intralaboratorial de métodos analíticos Determinação da repetibilidade e precisão intermediária.

2- Johnson \& Wichern (1998). Applied Multivariate Statistical Analysis, Fourth Edition, Prentice Hall.

3- Peternelli, L. A. ; Mello, M. P. (2011). Conhecendo o R - Uma visão mais que estatística; Editora UFV - 2013)

4- Hogg \& Tanis (1999). Probability and Statistical Inference, Fifth Edition, Prentice Hall.

5- Bussab, Wilton de O. e Morettin, Pedro A. (2011). Estatística Básica; 7ª̣ Edição; Editora Saraiva.

6- Conover, W. J. (1999). Practical Nonparametric Statistics, Third Edition, Wiley.

7- ISO 5725-2: (1994). Accuracy (trueness and precision) of Measurement Methods and Results. Part 6: Use in practice of accuracy values, International Organization for Standardization; Geneva; 2001.

8- Grubbs, Frank E (1969), Procedures for detecting outlying observations in samples, Taylor \& Francis Group, vol 11, p 1-21.

9- Montgomery, D. C. (1997), Design and Analysis of Experiments, Fourth Edition Ed. John Wiley \& Sons 